\title{
Fatty acid ethyl esters in virgin olive oils: A correlation study with the volatile profile
}

\author{
Giacomo Squeo, Graziana Difonzo, Vito. M. Paradiso, Carmine Summo, Antonella Pasqualone, \\ Francesco Caponio*
}

Department of Soil, Plant and Food Science, University of Bari, Via Amendola 165/A, 70126 Bari, Italy

\section{A B S T R A C T}

\begin{abstract}
Fatty acid ethyl esters are the newest mandatory quality parameters introduced for extra virgin olive oil classification linked to fermentative processes occurring in low-quality olives. Although it is clear the strong linkage of ethyl esters with sensory fermentative defects there is a lack of knowledge about their relationship with specific volatile compounds. Hence, a study was carried out to evaluate the correlation among extra virgin olive oil quality indices and its respective volatile profile with fatty acid ethyl esters. Thirty-five virgin olive oils were collected from local oily industry and analysed for the fatty acid ethyl esters content, the basic quality parameters, the total phenolic content and the volatile profile. The obtained results showed that the fatty acid ethyl esters were negatively correlated to total phenolic compounds $(p<0.05)$ and trans-2-hexenal content $(p<0.001)$ while positively correlated $(p<0.001)$ to ethyl acetate and ethyl acetate/trans-2-hexenal and ethyl acetate/hexanal ratios. Interestingly, ethanol was not correlated at all with the ethyl esters content in oils.
\end{abstract}

Keywords: Extra virgin olive oil; Fatty acid ethyl esters; Phenolic compounds; Volatile compounds

\section{INTRODUCTION}

Virgin olive oils are obtained directly from the fruits only by mechanical extraction (Official Journal of the European Communities, 2001). Only those oils that fulfil all the requirements established by the different regulations issued by the European Community can be classified in a specific category, such as extra virgin olive oil (EVOO) (Official Journal of the European Union, 2016). Good fruits and extraction practices are necessary conditions, but sometimes not sufficient, to obtain high quality oils. In fact, the final characteristics of a specific oil are dependent on pre-harvest as well as post-harvest factors (Mele et al., 2018). Among the former, genotype and ripeness degree strongly influence the phenolic and volatile amount and profile of the oil, as well as the basic chemical parameters such as free acidity which tended to rise, especially in damaged fruits (Conte et al., 2019; Giuffrè, 2018; Rotondi et al., 2010). Moreover, even soil, canopy position, and growing conditions/treatments could have a significant effect, as reported elsewhere (Caruso et al., 2017; Rached et al., 2017).
Among the analytical parameters of EVOO, the EU Regulation 61/2011 (Official Journal of the European Union, 2011) introduced for the first time the determination of the fatty acid alkyl esters (FAAEs), constituted by the sum of the fatty acids methyl esters (FAMEs) and ethyl esters (FAEEs), as quality parameter for the EVOO. Successively, the EU Regulation 1348/2013 (Official Journal of the European Union, 2013) focused only on the sum of FAEEs, which must not exceed $40 \mathrm{mg} \mathrm{kg}^{-1}$ in the $2013 / 14$ crop year, $\leq 35 \mathrm{mg} \mathrm{kg}^{-1}$ in the $2014 / 15$ harvest year and $\leq 30 \mathrm{mg} \mathrm{kg}^{-1}$ from the $2015 / 16$ crop year. Finally, the EU Regulation 2016/2095 (Official Journal of the European Union, 2016) set the maximum value of FAEEs for EVOO at $35 \mathrm{mg} \mathrm{kg}^{-1}$ of oil.

The presence of FAMEs and FAEEs in olive oils has been known since the end of the $60 \mathrm{~s}$ when were identified in stripping products (Bendini et al., 2009). Cert (2006) related the presence of such compounds to the fermentation phenomena that may occur in the olive fruit with formation of methyl and ethyl alcohols that react with free fatty acids (FFAs). Furthermore, the author studied the behaviour

\footnotetext{
*Corresponding author:

Francesco Caponio, Department of Soil, Plant and Food Science, University of Bari, Via Amendola 165/A, 70126 Bari, Italy.

E-mail: francesco.caponio@uniba.it
}

Received: 02 July 2019; Accepted: 30 August 2019 
of these esters to deodorization by applying two types of technology, under steam and under nitrogen, and found that up to $150{ }^{\circ} \mathrm{C}$ there is no loss of these products. Based on these results, Pérez-Camino et al. (2008) and Jabeur et al. (2015) used the alkyl esters of fatty acids to detect the addition of deodorized oils in EVOO. Alongside, Mariani and Bellan (2008) suggested these esters as quality markers, useful in detecting the EVOO produced from poor quality olives. The authors analysed more than 150 olive oils of various classes. It was found that alkyl esters in lampante oils were significantly abundant than in EVOOs, so that they could be eligible as an analytical tool to detect illegal mixture of lampante oil in EVOO, even those subjected to mild treatments in order to remove negative volatile compounds and bring them closer to the virgin. In good quality EVOO the presence of these esters was very low and, often, the FAMEs were higher than ethyl ones (PérezCamino et al., 2002). Other studies have also highlighted as ethyl and methyl esters of fatty acids in EVOO could be affected by the technological operations during the extraction process, while they were not affected by oil storage (Alcalá et al., 2017; Squeo et al., 2017a; Caponio et al., 2018a). As a conclusion, when appreciable amounts of alkyl esters of fatty acids in EVOO were found there was a high probability of illegal mixture of refined oils with analytical characteristics very similar to those of EVOO. Furthermore, other authors (Biedermann et al., 2008; Gómez-Coca et al., 2012) have shown a close correlation between chemical parameters and sensory evaluation; in particular, EVOOs, characterized by a low content of alkyl esters and a low content of waxes, were recognizer as high quality oils from the panel test, while those samples having high levels of FAAEs have not been judged as EVOOs. The FAAEs, in fact, have been reported to be strongly related with fermentative sensory defects such as winey, moldy and fusty-muddy (Gómez-Coca et al., 2012). As reported in several studies, both the sensory defect than the positive sensory attribute of the oils was strongly linked to the volatile profile of the oils (Cayuela et al., 2015; Kalua et al., 2007).

As far as we know, no investigations are available in literature in which the fatty acid ethyl esters in VOOs were correlated with the volatile profile of the same. In this framework, our aim was at studying the relationship between the total content of FAEE in virgin olive oils with the volatile profile and basic quality parameters.

\section{MATERIALS AND METHODS}

\section{Sampling}

One litre of thirty-five VOOs, packaged in green glass having about 3-mL of headspace, were collected by local oily industries in Apulia region in the months of November and December 2017. All the oils, produced by a two- or threephase centrifuge extraction system from drupes freshly harvested, upon arrival in the laboratory were subjected to analytical determinations and stored until completion of the analyses, about one month, in the dark at $4{ }^{\circ} \mathrm{C}$. Each sample was representative of a large batch of oil, extracted from different cultivars and ripeness degree of the fruits, sampled from tanks having a capacity of about 10 tons.

\section{Chemicals}

n-hexane, Folin-Ciocalteu reactive, gallic acid, methyl heptadecanoate and Sudan I dye were purchased from Sigma-Aldrich (Sigma-Aldrich Co. LLC, St. Louis, USA); diethyl ether and acetic acid were purchased from Honeywell (Honeywell International, Inc., Morristown, NJ, USA); ethanol, potassium iodide, sodium thiosulfate, chloroform, isooctane and methanol were purchased from Carlo Erba (CARLO ERBA Reagents S.r.l., Cornaredo, Milan, Italy); phenolphthalein solution was purchased from Fluka (Honeywell International, Inc., Morristown, NJ, USA); silica gel was purchase from Merck (Merck KGaA, Darmstadt, Germany).

\section{Analytical determinations}

Routine analyses (free acidity, peroxide value and UV spectrophotometric constants) were carried out according to EC Regulations (Official Journal of the European Communities, 1991).

The total phenolic compounds (TPCs) of the oils were extracted by liquid-liquid extraction using $5 \mathrm{~mL}$ of methanol/water (70:30, v/v) mixture and $1 \mathrm{~g}$ of sample and analysed as reported in Tamborrino et al. (2019) using the Folin-Ciocalteu reagent. The results were expressed as mg of gallic acid equivalent per $\mathrm{kg}$ of oil.

The FAEEs were determined as prescribed by the official analytical methods of EU Regulations (Official Journal of the European Union, 2011). Briefly, alkyl esters were firstly recovered by silica gel column chromatography and then measured by capillary gas chromatography. Each sample was analysed twice.

The volatile compounds analysis was carried out as reported in Caponio et al. (2018b) by SPME-GC-MS. Volatiles were extracted by means of a $50 / 30 \mu \mathrm{m}$ divinylbenzene/ carboxen/polydimethylsiloxane (DVB/CAR/PDMS) fiber (Supelco, Bellefonte, PA, USA) at $40^{\circ} \mathrm{C}$ for $20 \mathrm{~min}$ and then desorbed in the GC injector. The identification was carried out by comparison of their mass spectra and retention times with those of authentic reference compounds and the NIST and Wiley libraries. The results were expressed as total ion count. 


\section{Statistical analysis}

Minitab 17 software (Minitab Inc., State College, PA, USA) was used to carried out both the Principal Component Analysis (PCA) and the correlation analysis among variables and to calculate the respective significance $p$-values.

\section{RESULTS AND DISCUSSION}

Chemical characteristics of the analysed VOOs are showed in Fig. 1. In particular, i) FFAs mean value was $0.42{\mathrm{~g} 100 \mathrm{~g}^{-1}}^{-1}$ (range 0.19-2.28 g $100 \mathrm{~g} \mathrm{~g}^{-1}$ ) with one sample with values higher than the limit provided for EVOO; ii) PV mean value was 8.6 meq $\mathrm{O}_{2} \mathrm{~kg}^{-1}$ (range 1.8-23.1 meq $\mathrm{O}_{2} \mathrm{~kg}^{-1}$ ) with one sample with values higher than the limit provided for EVOO; iii) $\mathrm{K}_{232}$ mean value was 1.95 (range 1.18-3.54) with three samples exceeding the limit for EVOO; iv) $\mathrm{K}_{270}$ mean value was 0.22 (range 0.06-0.37) with eleven samples exceeding the limit for EVOO, among which were included those out of the limit for the other chemical parameters; v) FAEEs showed mean value of $8.01 \mathrm{mg} \mathrm{kg}^{-1}$ (range 2.51$20.13 \mathrm{mg} \mathrm{kg}^{-1}$ ) without samples with values exceeding the

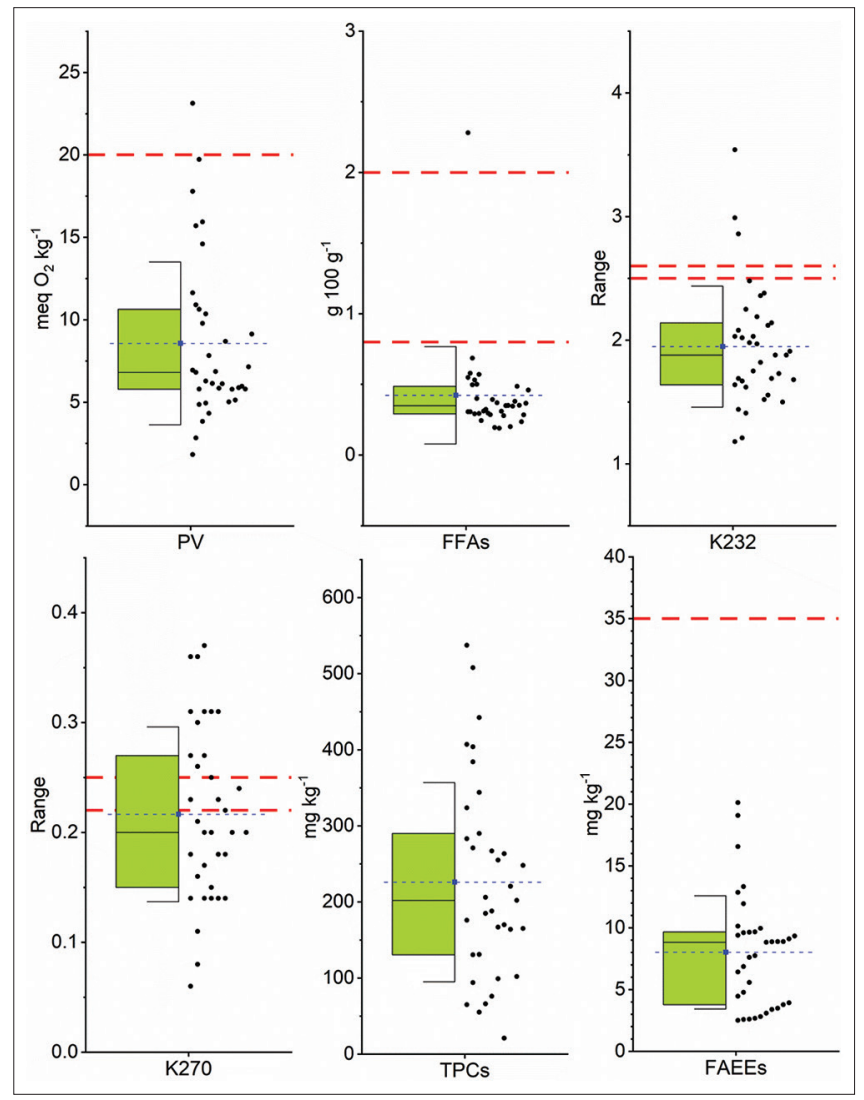

Fig 1. Chemical characteristics of the analysed VOOs. PV, peroxide value; FFAs, free fatty acids; $\mathrm{K}_{232}$ and $\mathrm{K}_{270}$, specific absorption at 232 and $270 \mathrm{~nm}$; TPCs, total polyphenol compounds; FAEEs, fatty acid ethyl esters. The dotted bars in red indicated the threshold for extra virgin (lower), virgin (higher) or both the oils, according to the Regulation EEC 2568/91. limit for EVOO. Moreover, TPCs showed mean values of $226 \mathrm{mg} \mathrm{kg}^{-1}$ (range 21-537 $\mathrm{mg} \mathrm{kg}^{-1}$ ).

The oils were also characterized by means of the analysis of volatile compounds and, as showed in Fig. 2, the aldehydes were the most abundant compounds.

More in detail, trans-2-hexenal - arising from linolenic acid oxidation (Zunin et al., 2004), and accountable for bitter and cut grass notes (Angerosa et al., 2004) - was the most abundant, in agreement with other authors (Aparicio et al., 1996; Angerosa et al., 2001; Caponio et al., 2014), representing on average more than $70 \%$ of the volatile fraction. It was followed by (a) trans-2-hexen1 -ol, having green and pungent notes, deriving from the reduction trans-2-hexenal by alcohol dehydrogenase (ADH) (Bedoukian, 1971; Morales et al.,1997); (b) hexanal, resulting from both enzymatic oxidation and autoxidation of linoleic acid (Zunin et al., 2004) and responsible for the sensory notes of green, apple, cut grass (Morales et al., 1997); (c) hexan-1-ol, originating from hexanal through the activity of $\mathrm{ADH}$ and responsible of fruity, aromatic, soft, cut grass notes (Angerosa et al., 2004); (d) ethanol, aring from fermentation and responsible for sensory notes of alcoholic, ripe apple, floral (Reiners and Grosch, 1998); (e) cis-3-hexen-1-ol and trans-trans-2,4-hexadienal, responsible for banana, leaf-like, green-fruity, pungent and cut grass notes, respectively (Angerosa et al., 2004); (f) ethyl-acetate, that may be synthesised through a different pathway (Salas, 2004; Kalua et al., 2007), responsible for sweet and aromatic notes (Morales et al., 1997). Both nonanal, deriving from autoxidation of oleic acid (Paradiso et al 2016, Paradiso et al., 2018a) and considered a marker of olive oil oxidation, and hexanoic acid, formed by oxidation of the corresponding aldehyde (Paradiso et al., 2018b), were present in very small amount, indicating a low incidence of the phenomenon of auto-oxidation in the examined samples.

PCA is a powerful multivariate exploratory analysis able to highlight underlying structure in the dataset and variables correlations. Fig. 3 shows the loading plot from PCA considering, as variables, the basic quality parameters, the TPC and, among the volatiles, trans-2-hexenal, hexanal, ethanol, and ethyl-acetate, in turn responsible for the characteristic positive flavour of EVOOs and for fermentative defects (Angerosa et al., 2004; Kalua et al., 2007). Moreover, also the relative amount of fermentation markers (ethanol (E) and ethyl-acetate (EA)) respect to the positive $\mathrm{C} 6$ aldehydes (trans-2-hexenal $(\mathrm{t} 2 \mathrm{H})$ and hexanal $(\mathrm{H})$ ) have been considered. The first two principal components, PC1 and PC2, explained together about 55\% of total variability. The plot evidenced that FAEEs were quite strongly and positively correlated with ethyl-acetate 


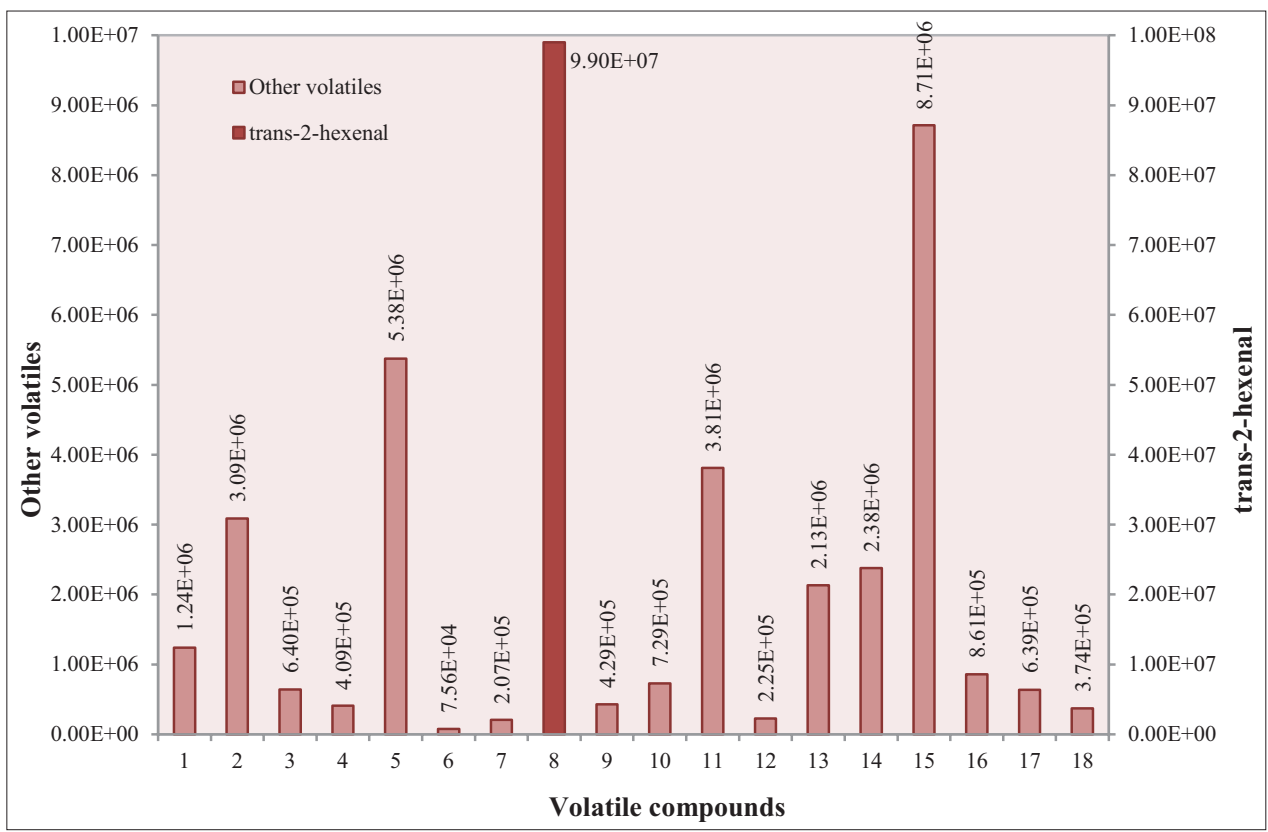

Fig 2. Mean values (total ion count of the integrated area) of the volatiles detected in the analysed VoOs. 1, ethyl acetate; 2 , ethanol; 3 , pentan3-one; 4, 1-penten-3-one, 5, hexanal; 6, 1-penten-3-ol; 7, limonene; 8, trans-2-hexenal; 9, 3-hexen-1-ol, acetate; 10, hexan-1-ol; 11, nonanal; 12, trans,trans-2,4-hexadienal; 13, cis-3-hexen-1-ol; 14, trans-2-hexen-1-ol; acetic acid; 17, benzaldehyde; 18, hexanoic acid.

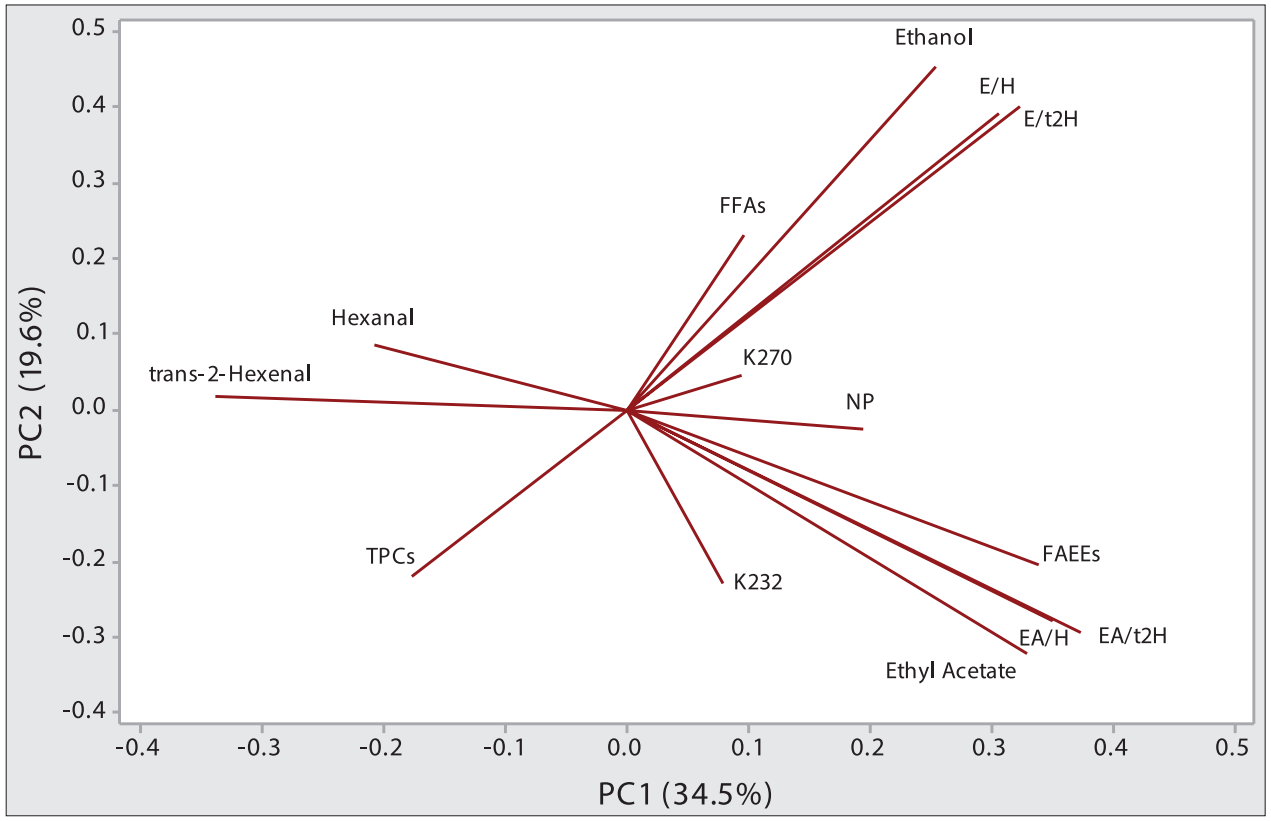

Fig 3. Loading plot from the principal component analysis (PCA) carried out on the selected data. PV, peroxide value; FFAs, free fatty acids; $\mathrm{K}_{232}$ and $\mathrm{K}_{270}$, specific absorption at 232 and $270 \mathrm{~nm}$; TPCs, total polyphenol compounds; FAEEs, fatty acid ethyl esters; EA, ethyl acetate; $\mathrm{E}$, ethanol; $\mathrm{t} 2 \mathrm{H}$, trans-2-hexenal; $\mathrm{H}$, hexanal.

rather than ethanol which, instead, seemed to be not correlated at all.

The result is noteworthy considering that ethanol is one of the precursors of fatty acids ethyl esters and thus a positive correlation would be reasonably expected. On the other hand, a negative correlation with the positive fruity compounds, trans-2-hexenal and hexanal, was observed.
The ratios $\mathrm{EA} / \mathrm{H}, \mathrm{EA} / \mathrm{t} 2 \mathrm{H}, \mathrm{E} / \mathrm{H}$, and $\mathrm{E} / \mathrm{t} 2 \mathrm{H}$ behaved very similar to the pure compounds being the $\mathrm{EA} / \mathrm{H}$ and EA/t2H strongly correlated with FAEEs. The Pearson's correlation coefficients were calculated along with the significance $p$-values. The results showed that a significant negative correlation existed between FAEEs and trans-2hexenal $(p<0.001)$ while, without statistical significance, among FAEEs and hexanal ( $p>0.05)$. Most important, a 
very significant negative correlation was found between ethyl esters and ethyl-acetate $(p<0.001)$ while the former were totally not correlated with ethanol, confirming what already observed through the PCA. Considering the indices $\mathrm{EA} / \mathrm{H}, \mathrm{EA} / \mathrm{t} 2 \mathrm{H}, \mathrm{E} / \mathrm{H}$, and $\mathrm{E} / \mathrm{t} 2 \mathrm{H}$, a strong correlation was found among FAEEs and $\mathrm{EA} / \mathrm{H}$ and $\mathrm{EA} / \mathrm{t} 2 \mathrm{H}$, being the former the most strongly correlated $(\mathrm{r}=0.746, p<0.001)$. Neither E/H nor E/t2H were correlated with FAEEs. As previously deducted from PCA, and confirmed by the Pearson's correlation, ethyl-acetate has been proven to be the most sensitive marker of the presence of fatty acids ethyl esters in the virgin olive oils under study. With regard to the TPC, a significant negative correlation with FAEEs was reported $(p<0.05)$. Phenolic content in oils depends on the ripeness degree and quality of the drupes as well as on technological procedures of olive paste preparation and oil extraction (Gómez-Caravaca et al., 2008; Alagna et al., 2012; Squeo et al., 2017b; Caponio et al., 2018b). Considering that ethyl esters are formed mainly as a consequence of fermentative processes occurring during prolonged and improper drupes storage, and, in turn, the same events cause the depletion of phenolic compounds, the correlation observed is reasonable. The same conclusions can be drawn considering the negative correlation of FAEEs with trans2-hexenal and hexanal. These findings confirmed that the processes responsible for the rising of ethyl esters in oils are linked together with a depletion of positive volatile and phenolic compounds as already known.

\section{CONCLUSION}

Our finding highlighted that FAEEs are strongly correlated with other analytical parameters of the EVOOs. In particular, FAEEs were significantly correlated in negative manner with TPCs and trans-2-hexenal and in positive manner with ethyl acetate and ethyl acetate/trans-2-hexenal and ethyl acetate/hexanal ratios.

\section{CONFLICT OF INTEREST}

The authors declare that there are no conflicts of interest regarding the publication of this paper.

\section{Author contributions}

Authors contributed equally to this study.

\section{REFERENCES}

Alagna, F., R. Mariotti, F. Panara, S. Caporali, S. Urbani, G. Veneziani, S. Esposto, A. Taticchi, A. Rosati, R. Rao, G. Perrotta, M. Servili and L. Baldoni. 2012. Olive phenolic compounds Metabolic and transcriptional profiling during fruit development. BMC Plant Biol. 12: 162-180.
Alcalá, S., M. T. Ocaña, J. R. Cárdenas, M. Á. Miquel, J. Vilar, F. Espínola and M. Moya. 2017. Alkyl esters content and other quality parameters in oil mill: A response surface methodology study. Eur. J. Lipid Sci. Technol. 119: 1600026.

Angerosa, F., M. Servili, R. Selvaggini, A. Taticchi, S. Esposto and G. F. Montedoro. 2004. Volatile compounds in virgin olive oil: Occurrence and their relationship with the quality. J. Chromatogr. A. 1054: 17-31.

Angerosa, F., R. Mostallino, C. Basti and R. Vito. 2001. Influence of malaxation temperature and time on the quality of virgin olive oils. Food Chem. 72: 19-28.

Aparicio, R., M. T. Morales and M. V. Alonso. 1996. Relationship between volatile compounds and sensory attributes of olive oils by the sensory wheel. J. Am. Oil Chem. Soc. 73: 1253-1264.

Bedoukian, P. Z. 1971. Seven primary hexenols and their olfactory characteristics. J. Agric. Food Chem. 19: 1111-1119.

Bendini, A., E. Valli, L. Cerretani, E. Chiavaro and G. Lercker. 2009. Study on the effects of heating of virgin olive oil blended with mildly deodorized olive oil: Focus on the hydrolytic and oxidative state. J. Agric. Food Chem. 57: 10055-10062.

Biedermann, M., A. Bongartz, C. Mariani and K. Grob. 2008. Fatty acid methyl and ethyl esters as well as wax esters for evaluating the quality of olive oils. Eur. Food Res. Technol. 228: 65-74.

Caponio, F., C. Summo, V. M. Paradiso and A. Pasqualone. 2014. Influence of decanter working parameters on the extra virgin olive oil quality. Eur. J. Lipid Sci. Technol. 116: 1626-1633.

Caponio, F., G. Squeo, L. Brunetti, A. Pasqualone, C. Summo, V. M. Paradiso, P. Catalano and B. Bianchi. 2018b. Influence of the feed pipe position of an industrial scale two-phase decanter on extraction efficiency and chemical-sensory characteristics of virgin olive oil. J. Sci. Food Agric. 98: 4279-4286.

Caponio, F., G. Squeo, M. Curci, R. Silletti, V. M. Paradiso, C. Summo, C. Crecchio and A. Pasqualone. 2018a. Calcium carbonate effect on alkyl esters and enzymatic activities during olive processing. Ital. J. Food Sci. 30: 381-392.

Caruso, G., R., Gucci, M. I., Sifola, R., Selvaggini, S., Urbani, S., Esposto, A. Taticchi and M. Servili. 2017. Irrigation and fruit canopy position modify oil quality of olive trees (cv. Frantoio). J. Sci. Food Agric. 97: 3530-3539.

Cayuela, J. A., R. B. Gómez-Coca, W. Moreda and M. D. C. PérezCamino. 2015. Sensory defects of virgin olive oil from a microbiological perspective. Trends Food Sci. Technol. 43: 227-235.

Cert, A. 2006. Meeting of Chemists to Study Methods of Analysis Olive Oils and Olive Pomace Oils. T20/Doc. No. 53-3. International Olive Council, Madrid, Spain.

Conte, P., G. Squeo, G. Difonzo, F. Caponio, C. Fadda, A. Del Caro, P. P. Urgeghe, L. Montanari, A. Montinaro and A. Piga. 2019. Change in quality during ripening of olive fruits and related oils extracted from three minor autochthonous Sardinian cultivars. Emir. J. Food Agric. 31: 196-205.

Giuffrè, A. M. 2018. The evolution of free acidity and oxidation related parameters in olive oil during olive ripening from cultivars grown in the region of Calabria, South Italy. Emir. J. Food Agric. 30: 539-548.

Gómez-Caravaca, A. M., L. Cerretani, A. Bendini, A. SeguraCarretero, A. Fernández-Gutiérrez, M. Del Carlo, D. Compagnone and A. Cichelli. 2008. Effects of fly attack (Bactrocera oleae) on the phenolic profile and selected chemical parameters of olive oil. J. Agric. Food Chem. 56: 4577-4583.

Gómez-Coca, R. B., W. Moreda and M. C. Pérez-Camino. 2012. Fatty acid alkyl esters presence in olive oil vs. organoleptic 
assessment. Food Chem. 135: 1205-1209.

Jabeur, H., A. Zribi, R. Abdelhedi and M. Bouaziz. 2015. Effect of olive storage conditions on Chemlali olive oil quality and the effective role of fatty acids alkyl esters in checking olive oils authenticity. Food Chem. 169: 289-296.

Kalua, C. M., M. S. Allen, D. R. Jr. Bedgood, A. G. Bishop, P. D. Prenzler and K. Robards. 2007. Olive oil volatile compounds, flavour development and quality A critical review. Food Chem. 100: 273-286.

Mariani, C. and G. Bellan. 2008. Detection of low quality oils in extra virgin olive oils. Riv. Ital. Sostanze Grasse 85: 3-20.

Mele, M. A., M. Z. Islam, H. M. Kang and A. M. Giuffrè. 2018. Preand post-harvest factors and their impact on oil composition and quality of olive fruit. Emir. J. Food Agric. 30: 592-603.

Morales, M. T., J. J. Rios and R. Aparicio. 1997. Changes in the volatile composition of virgin olive oil during oxidation: Flavors and off-flavors. J. Agric. Food Chem. 45: 2666-2673.

Official Journal of the European Communities. 1991. Council Regulation No. 2568/1991, L. 248 of July $11^{\text {th }}$, Publications Office of the European Union, Bruxelles.

Official Journal of the European Communities. 2001. Council Regulation No. 1513/2001, N. L. 201 of July $26^{\text {th }}$, Publications Office of the European Union, Bruxelles.

Official Journal of the European Union. 2011. Commission Regulation No. 61/2011, N. L. 23 of January $1^{\text {st }}$, Publications Office of the European Union, Bruxelles.

Official Journal of the European Union. 2013. European Community Regulation No. 1348/2013, N. L. 338 of December $17^{\text {th }}$, Publications Office of the European Union, Bruxelles.

Official Journal of the European Union. 2016. European Community Regulation No. 2095/2016, L. 326 of September $26^{\text {th }}$, Publications Office of the European Union, Bruxelles.

Paradiso, V. M., C. Di Mattia, M. Giarnetti, M. Chiarini, L. Andrich, and F. Caponio. 2016. Antioxidant behavior of olive phenolics in oil-in-water emulsions. J. Agric. Food Chem. 64: 5877-5886.

Paradiso, V. M., A. Pasqualone, C. Summo, and F. Caponio. 2018a. An "omics" approach for lipid oxidation in foods: The case of free fatty acids in bulk purified olive oil. Eur. J. Lipid Sci. Technol. 120: 1800102.

Paradiso, V. M., A. Pasqualone, C. Summo, and F. Caponio. 2018b.
Everything should be as simple as it can be. But not simpler. Does food lipid oxidation require an OMICS approach? Eur. J. Lipid Sci. Technol. 120: 1800103.

Pérez-Camino, M. C., A. Cert, A. Romero-Segura, R. Cert-Trujillo and W. Moreda. 2008. Alkyl esters of fatty acids a useful tool to detect soft deodorized olive oils. J. Agric. Food Chem. 56: 67406744.

Pérez-Camino, M. C., W. Moreda, R. Mateos, and A. Cert. 2002. Determination of esters of fatty acids with low molecular weight alcohols in olive oils. J. Agric. Food Chem. 50: 4721-4725.

Rached, M. B., G., Galaverna, M., Cirlini, D., Boujneh, M., Zarrouk and M. Guerfel. 2017. Pedologic factors affecting virgin olive oil quality of "Chemlali" olive trees (Olea europaea L.). J. Oleo Sci. 66: 907-915.

Reiners, J. and W. Grosch. 1998. Odorants of virgin olive oils with different flavor profiles. J. Agric. Food Chem. 46: 2754-2763.

Rotondi, A., B., Alfei, M., Magli and Pannelli, G. 2010. Influence of genetic matrix and crop year on chemical and sensory profiles of Italian monovarietal extra-virgin olive oils. J. Sci. Food Agric. 90: 2641-2648.

Salas, J. J. 2004. Characterization of alcohol acyltransferase from olive fruit. J. Agric. Food Chem. 52: 3155-3158.

Squeo, G., A. Tamborrino, A. Pasqualone, A. Leone, V. M. Paradiso, C. Summo and F. Caponio. 2017b. Assessment of the influence of the decanter set-up during continuous olive oil processing at different pigmentation index. Food Bioprocess Techn. 10: 592-602.

Squeo, G., R. Silletti, C. Summo, V. M. Paradiso, A. Pasqualone and F. Caponio. 2017a. Fatty acids methyl and ethyl esters behaviour during olives processing by means of technological coadjuvants. Ital. J. Food Sci. 29: 370-376.

Tamborrino, A., R. Romaniello, F. Caponio, G. Squeo and A. Leone. 2019. Combined industrial olive oil extraction plant using ultrasounds, microwave, and heat exchange: Impact on olive oil quality and yield. J. Food Eng. 245: 124-130.

Zunin, P., R. Boggia, S. Lanteri, R. Leardi, R. De Andreis and F. Evangelisti. 2004. Direct thermal extraction and gas chromatographic mass spectrometric determination of volatile compounds of extra-virgin olive oils. J. Chromatogr. A. 1023: 271-276. 